\title{
No high-level evidence to support use of ultrasonic instrumentation for root canal treatment
}

\author{
Abstracted from \\ Pedrazzi V, Oliveira-Neto JM, Sequeira P, Fedorowicz Z, Nasser M. \\ Hand and ultrasonic instrumentation for orthograde root canal treatment of permanent teeth. \\ Cochrane Database Syst Rev 2008; issue 4 \\ Address for correspondence: Vinícius Pedrazzi, Departamento de Materiais Dentários e Prótese, \\ Faculdade de Odontologia de Ribeirão Preto, Universidade de São Paulo, Avenida do Café s/no, \\ Ribeirão Preto, São Paulo, 14040-904, Brazil. E-mail: pedrazzi@forp.usp.br
}

\section{Question: For orthograde root canal treatment of permanent teeth, is hand instrumentation more effective than ultrasonic instrumentation when used alone or as an adjunctive procedure?}

Data Sources Searches were made for suitable reports using the Cochrane Oral Health Group Trials Register, Cochrane Central Register of Controlled Trials, Medline, Embase and LILACS (Latin American and Caribbean Health Sciences Literature), and within the reference lists of identified articles. There were no language restrictions. Study selection Randomised controlled trials (RCT) were selected if they had participants of over 18 years of age who had single and multiple permanent teeth with a completely formed apex and with no evidence of internal resorption, and which required root canal treatment. Individuals undergoing retreatment of a tooth were excluded. Data extraction and synthesis Screening of eligible studies was conducted in duplicate and independently. Results were to be expressed as fixed-effect or random-effects models using mean differences for continuous outcomes and risk ratios for dichotomous outcomes with $95 \%$ confidence intervals. Heterogeneity was to be investigated including both clinical and methodological factors. Results No eligible RCT were identified.

Conclusions This review illustrates the current lack of published or ongoing RCT, and the lack of availability of high-level evidence dealing with clinically relevant outcomes, for the effectiveness of ultrasonic instrumentation used alone or as an adjunct to hand instrumentation for orthograde root canal treatment. Future RCT might focus more closely on evaluating the effectiveness of combinations of these interventions with an emphasis not only on clinically relevant but also patient-centred outcomes.

\section{Commentary}

There is no doubt that mechanical preparation of root canals plays a significant role in endodontic therapy. Current literature provides conflicting results for biomechanical efficiency for manual, rotary and ultrasonic instrumentation techniques. The question addressed by this review is a valid one, with ultrasonic instrumentation attracting significant interest lately because of the observations that these tools can produce smooth, progressively tapered canals regardless of root canal curvatures and their noticeable advantages when debriding and disinfecting the root canal system.

Although this review found no eligible RCT to help answer this question, other experimental reports showed no significant differences between ultrasonic and hand techniques in cleaning root canals. ${ }^{1-3}$ Combining instrumentation techniques with ultrasound technology, however, has been shown to be more efficient in eradicating biofilms. ${ }^{4}$ Therefore, until the results of future RCT are published, the current belief is that ultrasonic application in conjunction with other instrumentation techniques does reduce microbial load but that any greater efficiency of ultrasonic instrumentation alone has yet to be demonstrated when obtaining the mechanical objectives of root canal preparation.

\section{Practice points}

- Based on clinically relevant outcomes, there is no evidence to support the superiority of ultrasonic instrumentation over hand instrumentation.

- Experimental, relevant outcomes do show the superiority of combining both techniques in terms of biological preparation.

Khaled Balto

Faculty of Dentistry, King Abdulaziz University, Jeddah, Saudi Arabia

1. Walker TL, del Rio CE. Histological evaluation of ultrasonic and sonic instrumentation of curved root canals. J Endod 1989; 15: 49-57

2. Cymerman JJ, Jerome LA, Moodnik RM. A scanning electron microscope study comparing the efficacy of hand instrumentation with ultrasonic instrumentation of the root canal. J Endod 1983; 9: 327-331.

3. Targ MPF, Stock JR. The effect of hand, sonic and ultrasonic instrumentation on the shape of curved root canals. Int Endod J 1989; 22: 55-63.

4. Sabins RA, Johnson JD, Hellstein JW. A comparison of the cleaning efficacy of short-term sonic and ultrasonic passive irrigation after hand instrumentation in molar root canals. J Endod 2003; 29: 674-678

Evidence-Based Dentistry (2009) 10, 76. doi:10.1038/sj.ebd.6400668 